\title{
The Role of Attached Phase Soil and Sediment Organic Matter Physicochemical Properties on Fullerene $\left(\mathrm{nC}_{60}\right)$ Attachment
}

\author{
Coy P. McNew ${ }^{\mathrm{a}}$, Eugene J. LeBoeuf ${ }^{\mathrm{a}, *}$ \\ ${ }^{a}$ Department of Civil and Environmental Engineering, Vanderbilt University, Nashville, \\ Tennessee 37235, United States
}

\section{Abstract}

Attached phase soil and sediment organic matter is ubiquitous in the subsurface environment, with a tendency to strongly sorb contaminants, and therefore it may play an important role in contaminant transport. In this study, the deposition of $\mathrm{C}_{60}$ nanoparticles onto attached phase Harpeth $\mathrm{Hu}-$ mic Acid and Harpeth Fulvic Acid (HHA and HFA) is explored by using a quartz crystal microbalance with dissipation monitoring and systematically varying thermal energy. By comparing the $\mathrm{C}_{60}$ attachment onto HHA and HFA surfaces to that of bare silica and DLVO predictions, we find that the HHA and HFA layers hinder attachment at low temperatures, while HHA enhances attachment at higher temperatures. Based on thermal characterization of the HHA and HFA layers compared to the corresponding attachment trends, the attachment efficiency is strongly correlated with hydration of the layer. Possible mechanisms explaining this phenomenon include water-

\footnotetext{
${ }^{*}$ Corresponding author

Email addresses: coy.p.mcnew@vanderbilt.edu (Coy P. McNew), eugene.j.leboeuf@vanderbilt.edu (Eugene J. LeBoeuf)
} 
assisted disruption of polar SOM contacts and hydration-induced swelling of the AP-SOM matrix. Since humic substances typically dominate subsurface organic matter, these results may prove crucial to understanding the complex interactions of engineered nanomaterials in both the natural and engineered environment.

Keywords: fullerene, "C60", humic acid, fulvic acid, QCM-D, SOM

\section{Introduction}

2

In 2014, the nanotechnology market was valued at around $\$ 26$ billion and it is expected to grow to $\$ 64.2$ billion over the next five years (McWilliams, 2014). In particular, the buckminsterfullerene $\left(\mathrm{C}_{60}\right)$ molecule has attracted much attention due to its widespread applications in many areas (e.g. biomedical technology, electronics, and optics) (Mauter and Elimelech, 2008; Bosi et al., 2003, Nel et al., 2006). With such a high level of production and dissemination, the eventual release of $\mathrm{C}_{60}$ into the environment is inevitable, either from industrial sources or through disposal in municipal waste. Several studies have investigated and revealed the potential human health effects, aquatic toxicity, and antimicrobial properties of $\mathrm{C}_{60}$ (Nel et al., 2006; Kamat et al., 2000; Lyon et al., 2006; Nakajima et al., 1996). The ongoing environmental release coupled with a strong potential for toxicity raise serious concern for the fate and transport of this material in the environment.

The fate and transport of $\mathrm{C}_{60}$ is mainly influenced by aggregation and deposition processes, which are controlled by a complex balance of variables including $\mathrm{pH}$, ionic strength, and presence of organic matter (Lin et al., 2010). Once in the environment, $\mathrm{C}_{60}$ will interact with natural soils and sediments, 
and thus, organic matter attached on the soil and sediment surfaces. Attached phase soil and sediment organic matter (AP-SOM) is the product of on-site physical and biological decomposition and is an essential component of natural soils and sediments. AP-SOM has been found to strongly sorb hydrophobic organic chemicals (HOCs) and thus influence their transport (Weber et al., 2001). If similar interactions between AP-SOM and $\mathrm{C}_{60}$ exist, these interactions will influence potential pathways of exposure and their eventual impact on ecosystems and human health.

Humic substances, including humins, humic acids, and fulvic acids, typically represent the dominant organic components in soils and sediments (Stevenson, 1994). Chemically, humic substances are a mixture of both substituted aromatic and aliphatic compounds, forming a complex physical structure held together by inter- and intra-molecular interactions (Sutton and Sposito, 2005) and therefore represent complex mixtures of macromolecules with varying degrees of crosslinkages. An important factor when considering the macromolecular properties of SOM is, of course, the presence of water. The hydration of SOM has been shown to alter the SOM matrix in several key ways, namely through altered physicochemical properties. Water has been shown to interact strongly with hydrophilic SOM, including fulvic and humic acids, resulting in water uptake and swelling of the macromolecular matrix (LeBoeuf and Weber, 1997; LeBoeuf, 1998). The physicochemical changes in SOM due to increased hydration have also been shown to significantly increase the sorption of HOCs (Graber and Borisover, 1998; Borisover et al., 2001; Borisover and Graber, 2002; Graber et al., 2007). The mechanism for increased sorption of HOCs due to increased hydration is likely 
explained by water-assisted disruption of polar SOM contacts, as described by Graber and Borisover (1998). As hydration increases, water molecules penetrate the SOM matrix and reach polar areas that were previously unavailable to HOC interaction, due to inter- or intra-molecular interactions. By hydrating these areas, the water molecules disrupt the interactions between these contacts and provide a new sorption site, which was previously unavailable. The extent to which this sorption enhancing characteristic of SOM hydration may apply to the deposition behavior of $\mathrm{C}_{60}$, however, has not yet been investigated.

Dissolved organic matter (DOM), in the form of humic substances, has been shown to adsorb to the surface of $\mathrm{C}_{60}$ aggregates and, through steric repulsion, increase their stability to aggregation (Chen and Elimelech, 2007) and deposition (Chen and Elimelech, 2008). Until very recently, however, little work has been done to investigate the interaction between $\mathrm{C}_{60}$ and the other form of ubiquitous SOM, AP-SOM. In recent studies, AP-SOM has been shown to enhance, hinder, or have no effect on the attachment of $\mathrm{C}_{60}$ depending on the type of AP-SOM and the ionic strength of solution (Chen and Elimelech, 2008; Qu et al., 2012). Furthermore, depending on the stabilizer, the transport of carbon nanotubes through humic acid coated quartz sand grains has been shown to vary between complete retention and minimal retention (Lu et al., 2014). Due to the variable nature of conclusions presented in these studies, it is clear that AP-SOM will play a complex role in the deposition of $\mathrm{C}_{60}$, likely dependent upon the type and physicochemical properties of AP-SOM and the ionic strength of solution.

The purpose of this study is to further investigate the role AP-SOM plays 
on the deposition of $\mathrm{C}_{60}$ by varying the type and physicochemical properties of AP-SOM. Using a quartz crystal microbalance with dissipation monitoring (QCM-D), the deposition of $\mathrm{C}_{60}$ onto silica surfaces with and without coatings of humic and fulvic acid was studied, while altering the physicochemical properties of the layer by varying temperature. The attachment efficiencies of $\mathrm{C}_{60}$ onto three different surfaces as a function of temperature are then compared to DLVO theory predictions, and the effect of AP-SOM physicochemical properties on the transport of $\mathrm{C}_{60}$ is discussed. Not only will knowledge of AP-SOM and engineered nanomaterial (ENM) interactions improve the understanding of the fate and transport of ENMs in the natural environment, but it will also aid in the engineered environment, through improved understanding of factors influencing the deposition behavior of similar types of ENMs for applications in water and wastewater treatment.

\section{Materials and Methods}

\subsection{Materials and solution chemistry}

Sublimed $\mathrm{C}_{60}$ powder (99.9\%) was purchased from MER Corporation (Tucson, AZ). Dry Harpeth River Humic Acid (HHA) and Fulvic Acid (HFA) were isolated as described in our previous publication (DeLapp et al., 2005). Sodium phosphate monobasic (S5011), sodium phosphate dibasic (S5136), and poly-L-lysine hydrobromide (PLL, P1274) were purchased from SigmaAldrich (St. Louis, MO). Reagent grade $\mathrm{NaCl}$ (S671) was purchased from Fisher Scientific (Pittsburgh, PA). Silica-coated QCM-D sensors (QSX-303) were purchased from Q-Sense (Biolin Scientific, Stockholm, Sweden). All solutions were prepared using ultra pure water purified by the Milli-Q Water 
Purification system from Millipore (Billerica, MA).

Sodium phosphate monobasic and sodium phosphate dibasic were mixed on a $50 \%$ molar basis to produce a phosphate buffer that would maintain all solutions at a $\mathrm{pH}$ of $6.7 \pm 0.1$. The background electrolyte concentration for all experiments consisted of $70 \mathrm{mM} \mathrm{NaCl}$ and $2 \mathrm{mM}$ of the previously described phosphate buffer.

\section{2. $C_{60}$ stock suspension and SOM Stock Solutions}

The $\mathrm{C}_{60}$ stock suspension was prepared using a method described by Andrievsky et al. (1995) (details of the preparation method are provided in Appendix A.1). The resulting $\mathrm{C}_{60}$ stock suspension was determined to have a total carbon content (Shimadzu TOC-V CPH/CPN, Columbia, MD) of $30.48 \mathrm{mg} / \mathrm{L}$ and an unadjusted $\mathrm{pH}$ of 5.5. The suspension was stored in the dark at $4{ }^{\circ} \mathrm{C}$ for the entirety of the study. All experiments took place within 3 months of preparing the $\mathrm{C}_{60}$ stock suspension, during which time the particle size was stable, as confirmed by periodic Dynamic Light Scattering (DLS) monitoring (Malvern Zetasizer Nano ZS, Worcestershire, UK).

The method for preparing the HHA and HFA solutions, used to build the AP-SOM layers, are provided in Appendix A.2. Using DLS, the intensity weighted hydrodynamic diameter of the HHA and HFA particles in solution was determined to be 247 and $390 \mathrm{~nm}$, respectively. Also using DLS, the zeta potential of the HHA and HFA particles were determined to be -43 and -12 $\mathrm{mV}$, respectively, based on 14 independent measurements. Both solutions were stored at room temperature in the dark for the entirety of the study, during which time the particle size was stable, as confirmed by periodic DLS monitoring. The HHA and HFA used in this study were characterized 
in a previous publication by DeLapp et al. (2005) and the elemental and functional group analysis are provided in Tables B.1 and B.2 Appendix B.1). Further characterization of the HHA and HFA layers can be found in Appendix B.1.

\section{3. $C_{60}$ deposition experiments}

The deposition of $\mathrm{C}_{60}$ nanoparticles onto silica surfaces with and without HHA and HFA coatings was explored using a Quartz Crystal Microbalance with Dissipation Monitoring (QCM-D, Q-Sense E4 unit, Biolin Scientific, Stockholm, Sweden). The unit consists of 4 flow modules, each containing a $5 \mathrm{MHz}$ AT-cut quartz crystal sensor. Flowrates were $0.1 \mathrm{~mL} / \mathrm{min}$, ensuring laminar flow within the flow module. Temperatures of the solution and flow module were maintained at $\pm 0.02{ }^{\circ} \mathrm{C}$ by a Peltier element.

Before each experiment, the silica coated quartz sensors (QSX303) were soaked in a $2 \%$ Hellmanex II solution overnight, rinsed with copious amounts of deionized water, dried with ultrapure $\mathrm{N}_{2}$ gas, and then cleaned in a UVOzone cleaner (Jelight 42 UVO Cleaner, Irvine, CA) for 20 minutes. Immediately after cleaning, the sensors were loaded into the QCM-D for execution of the experiment.

A representative $\mathrm{C}_{60}$ deposition experiment protocol is illustrated in Fig. 1. with detailed description provided in Appendix A.3. As $\mathrm{C}_{60}$ deposited onto the sensor surface, the mass continually increased, causing a continuous decrease in frequency, as described by the Sauerbrey Relation (Sauerbrey, 1959) (Eq. 1), where $m$ is the mass of material deposited, $C$ is the crystal constant $\left(17.7 \mathrm{ngcm}^{-2} \mathrm{~Hz}^{-1}\right)$, and $f_{n}$ is the frequency at overtone $n$. The first 120 seconds of frequency drop corresponding with $\mathrm{C}_{60}$ deposition were considered 
in calculating the initial deposition rate at each experimental condition.

$$
\Delta m=-C \frac{1}{n} \Delta f_{n}
$$

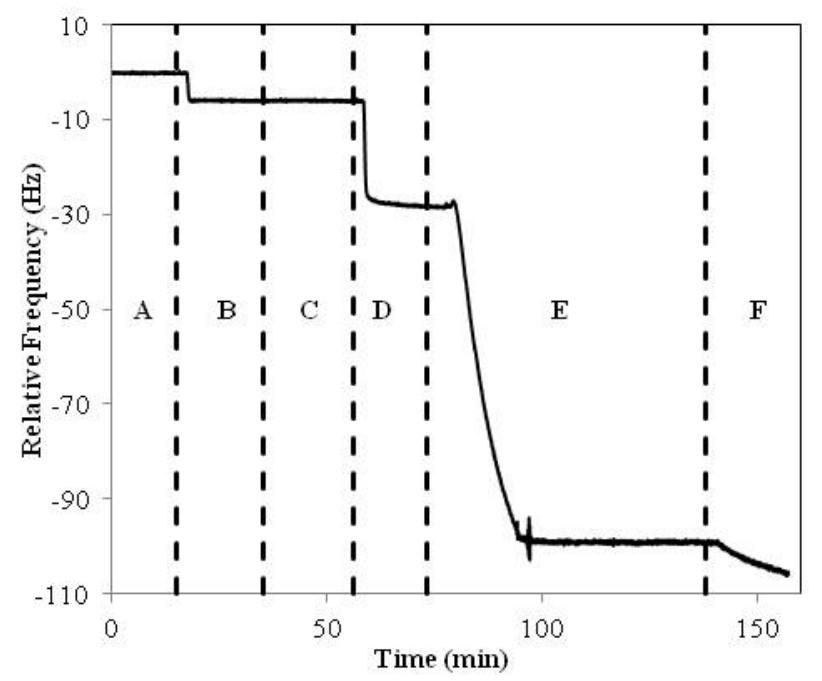

Figure 1: Representative $\mathrm{C}_{60}$ deposition experiment. The initial baseline was collected in the background solution (A) before PLL was attached to the silica surface (B). The attached PLL was then washed in the background solution (C) before HHA was attached to the PLL surface (D). The attached HHA was then washed with background solution and the temperature of the sensor and chamber was lowered to $15{ }^{\circ} \mathrm{C}(\mathrm{E})$. Once a stable baseline was observed at the new temperature, the $\mathrm{C}_{60}$ nanoparticle suspension with background solution was introduced and attached to the HHA surface (F).

144 While $\mathrm{C}_{60}$ deposited onto the sensor surfaces, the first 120 seconds of the shift in the third overtone of frequency $\left(f_{3}\right)$ were used to calculate the initial deposition rates. In order to compare deposition rates at different experimental conditions independent of $\mathrm{C}_{60}$ aggregation effects Chen and Elimelech, 2006), the deposition rates were normalized by calculating the attachment efficiency factor $(\alpha)$. The attachment efficiency factor was calculated (Eq. 
2) by normalizing the initial deposition rates to the corresponding initial deposition rate under favorable (nonrepulsive) conditions. Here, the favorable deposition rates are provided by the deposition rates of $\mathrm{C}_{60}$ onto oppositely charged PLL layers at identical experimental conditions.

$$
\alpha=\frac{d f_{3} / d t}{\left(d f_{3} / d t\right)_{f a v}}
$$

\section{Results and Discussion}

\section{1. $C_{60}$ nanoparticle suspension}

Investigation by Transmission Electron Microscopy (TEM) (Philips CM 20 TEM, Eindhoven, Netherlands) suggested the particles were polydisperse, as seen in Fig. 2, where representative images of an aggregate (left image) and individual particles (right image) are shown. DLS measurements confirmed a polydisperse particle distribution with an intensity weighted hydrodynamic diameter of the $\mathrm{C}_{60}$ nanoparticles of $103 \mathrm{~nm}$ based on 14 independent measurements and a zeta potential of $-42 \mathrm{mV}$, based on 20 independent measurements. The zeta potential of the suspension was measured over a $\mathrm{pH}$ range of $3-10$ and the results remained negative over the entire $\mathrm{pH}$ range investigated. Figures B.10 and B.11 Appendix B.2 summarize particle size distribution and zeta potential measurements.

\section{2. $C_{60}$ attachment analysis}

The deposition behavior of $\mathrm{C}_{60}$ onto bare silica has been shown to be in qualitative agreement with DLVO theory (Chen and Elimelech, 2006), therefore the total interaction energy profiles for $\mathrm{C}_{60}$ approaching a bare 


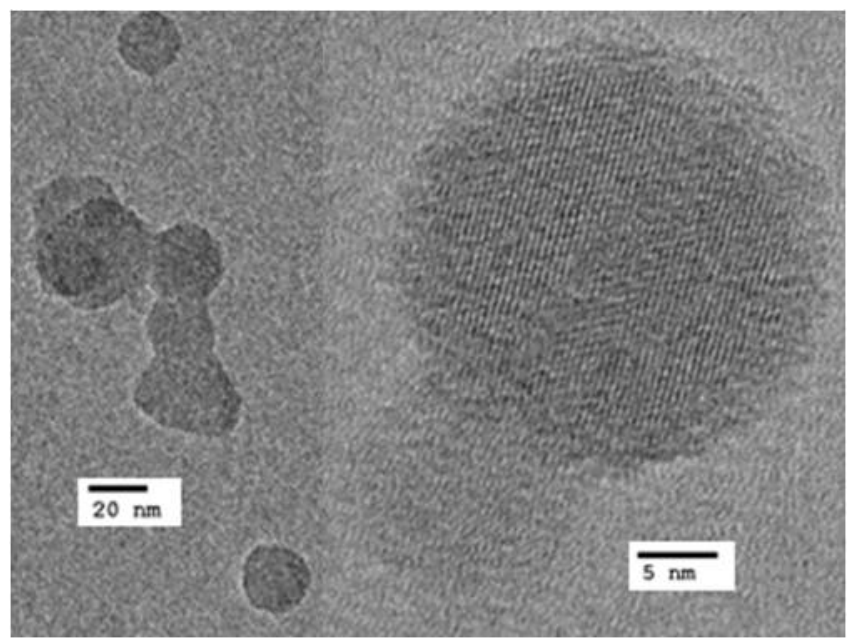

Figure 2: Representative TEM images of the $\mathrm{C}_{60}$ nanoparticle suspension.

silica surface were calculated for each temperature investigated and presented in Fig. 3. The detailed DLVO calculations are presented in Appendix C. As temperature increases, the total interaction energy profiles remain very similar, except for a small decrease in the energy barrier.

For the first time, the attachment efficiency factors of $\mathrm{C}_{60}$, onto three different surfaces, as a function of temperature are presented in Fig. 4. The bare silica attachment trend appears rather flat, with a small net increase, though the total increase does not fall outside the error of the measurements. This can be considered relatively consistent with DLVO predictions (Fig. 3), as the energy barrier shows a small decrease with increasing temperature, which could lead to a negligible increase in attachment. The presence of HHA and HFA coatings cause significant deviations from the bare silica trend. Compared to the attachment onto bare silica, both HHA and HFA hinder $\mathrm{C}_{60}$ attachment at lower temperatures, while HHA enhances attachment at higher temperatures. At the highest temperature investigated, HHA 


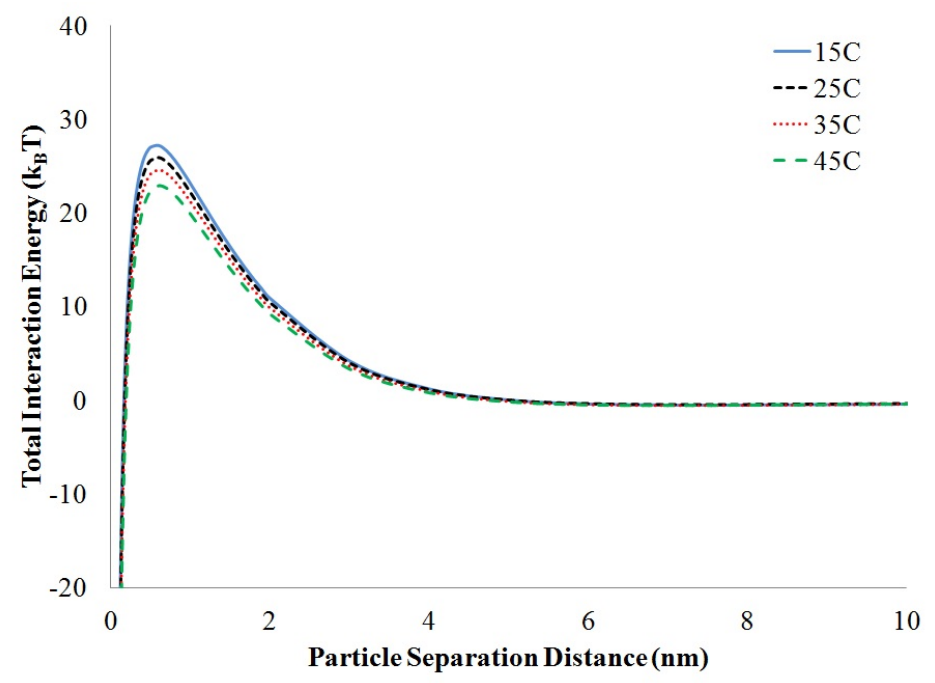

Figure 3: Calculated DLVO interaction energy profiles as $\mathrm{C}_{60}$ approaches a bare silica surface.

greatly enhances attachment while HFA appears to marginally enhance it, including a very slight overlap of error bars with the bare silica curve. The HHA and HFA trends continuously increase with increasing temperatures, though the rate of increase appears complex, presumably due to the change in physicochemical properties of the SOM layers over the temperature range investigated. The HHA trend displays a rate increase throughout the temperature range investigated, while the HFA trend appears to level off and then recover. The largest increase in attachment for both surfaces occurs in the temperature range of $35-45^{\circ} \mathrm{C}$. This suggests that the AP-SOM layers undergo the largest change in physicochemical properties in this region. Previous studies of the HHA and HFA have illustrated thermal transitions within this region (DeLapp et al., 2005), however these studies investigated the materials as a dry, bulk powder. In our current work, the HHA and 


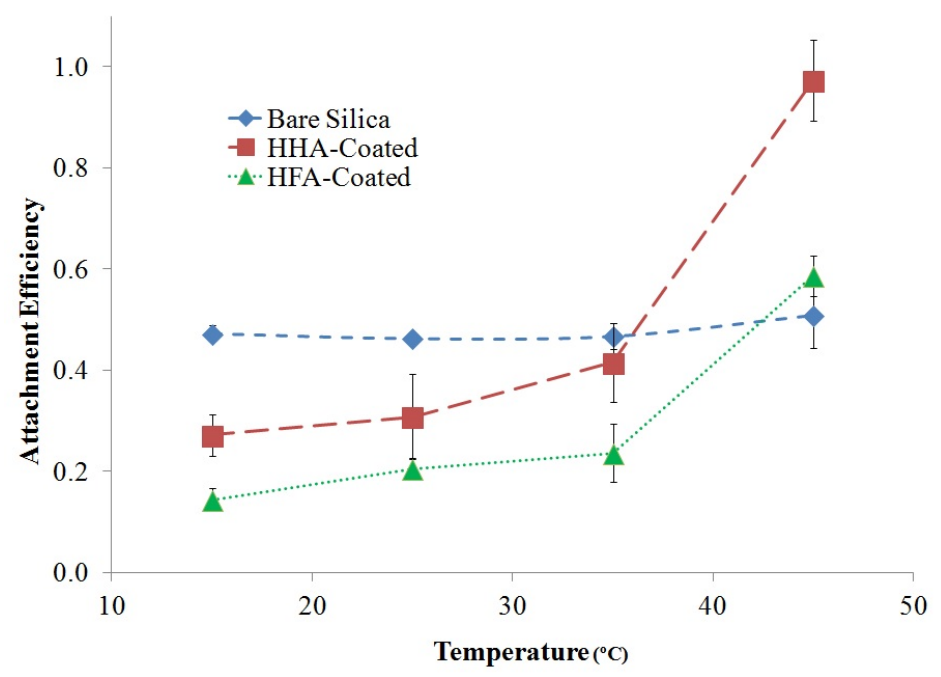

Figure 4: $\mathrm{C}_{60}$ attachment efficiencies onto bare, HHA-coated, and HFA-coated silica sensors as a function of temperature. Error bars represent the standard deviation.

HFA are examined as thin, hydrated layers, bringing into account additional variables and, because of this, the degree to which these studies apply to the current work is unknown.

\subsection{Thermal effects on attachment}

In order to understand the deviation in attachment onto the HHA and HFA surfaces, the thermal properties of the SOM layers were investigated using the QCM-D. This is a particularly novel approach because the QCMD has never before been used to thermally characterize AP-SOM. First, each AP-SOM layer was constructed following the same procedure as executed for the deposition experiments and as described previously. Then, the layer was exposed to a temperature ramp covering the entire temperature range of this study. The density and viscosity of water are a function of temperature, so the response of the sensor submerged in a solution is also expected to 


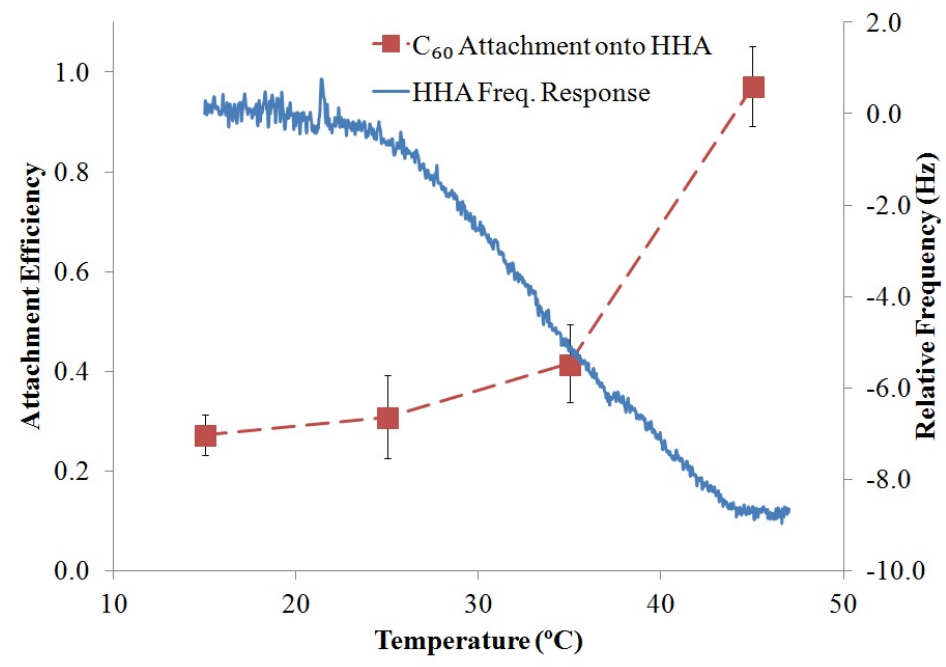

Figure 5: Thermal characterization of the HHA layer by QCM-D, with all bulk solution and sensor effects subtracted, compared to the corresponding $\mathrm{C}_{60}$ attachment efficiency trend. The 3rd overtone of frequency is displayed.

change as a function of temperature (Ishida and Biggs, 2007). In order to isolate the effects caused only by changes in each AP-SOM layer, the same bare sensor was first taken through an identical temperature ramp and its response was subtracted. In this manner, the QCM-D was utilized to thermally characterize a thin layer of AP-SOM. The frequency curves displayed in Figs. 5 and 6 represent the response of the HHA and HFA layers, respectively, to a change in temperature. By comparing the AP-SOM frequency response to the corresponding $\mathrm{C}_{60}$ attachment trend, we can gain insights into the mechanisms by which the attachment onto each AP-SOM deviates from that of bare silica.

The frequency curve provides insight into the mass changes of the layer, including any water molecules that are hydrating the layer. The overall trend 


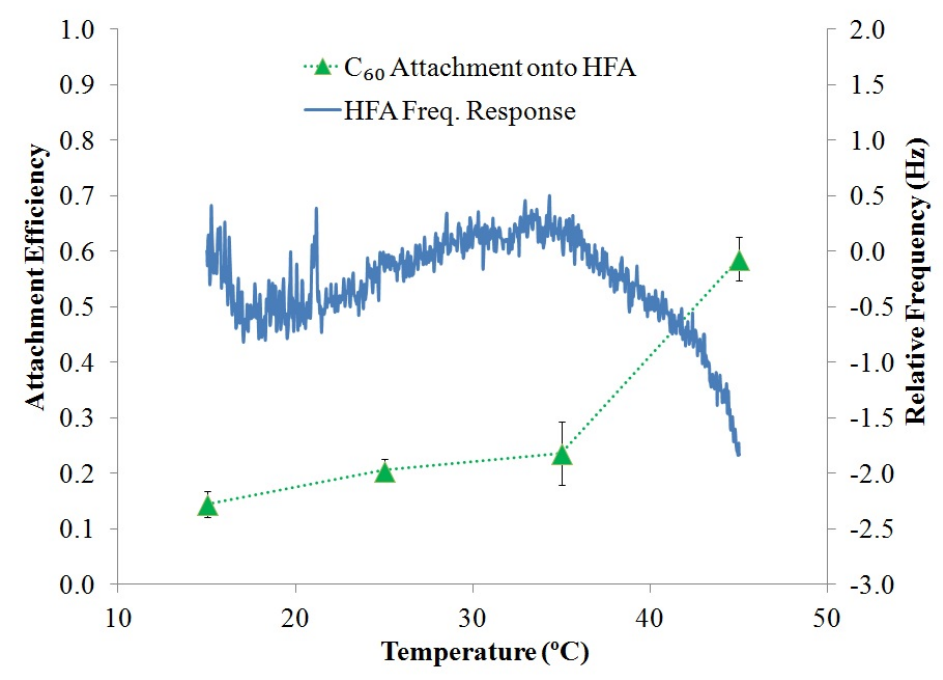

Figure 6: Thermal characterization of the HFA layer by QCM-D, with all bulk solution and sensor effects subtracted, compared to the corresponding $\mathrm{C}_{60}$ attachment efficiency trend. The 3rd overtone of frequency is displayed.

of each frequency curve is downwards, indicating an increase in mass (see Eq. 1), attributable to the hydration of the AP-SOM layer at increasing temperatures. The observed increase in hydration with increasing temperature is in agreement with a study by Vidyasagar et al. (2012), which investigated the thermal characteristics of a polyectrolyte using a similar QCM-D method. As indicated by Figs. 5 and 6, an increase in hydration (decrease in frequency) corresponds with an increase in attachment for both AP-SOM layers investigated. As the HHA layer begins to hydrate between $15-25^{\circ} \mathrm{C}$, the attachment shows a small increase (Fig. 5). As the HHA continues to hydrate more rapidly between $25-35$ and $35-45{ }^{\circ} \mathrm{C}$, the attachment also results in a sharper increase. As the HFA layer begins to hydrate in the range of 15-25 ${ }^{\circ} \mathrm{C}$, the attachment begins to rise as well (Fig. 6). As the HFA hydration 
rate slows and begins to reverse in the range of $25-35{ }^{\circ} \mathrm{C}$, however, the increase in attachment slows and begins to level off. The lack of an observable decrease in attachment in this region suggests that the interaction is a complex one, relying on more than just hydration of the layer. As the HFA layer resumes a rapid hydration rate between $35-45{ }^{\circ} \mathrm{C}$, the attachment shows a correspondingly rapid increase. For these reasons, Figs. 5 and 6 clearly illustrate a strong correlation between AP-SOM layer hydration and resulting $\mathrm{C}_{60}$ attachment efficiency, which, to our knowledge, has not been illustrated for ENMs.

The $\mathrm{C}_{60}$ suspension was also thermally characterized throughout the temperature range of $15-55^{\circ} \mathrm{C}$. The particle diameter was found to negligibly increase from $99 \mathrm{~nm}$ to $106 \mathrm{~nm}$ and the zeta potential showed no discernible change. Summaries of these measurements can be found in Figs. B.12 and B.13 Appendix B.2, respectively.

An increase in hydration of the AP-SOM layers at higher temperatures contribute to the increased attachment behavior through water-assisted disruption of polar SOM contacts, as described by Graber and Borisover (1998). As hydration increases, water molecules penetrate the SOM matrix and reach polar areas that were previously unavailable to $\mathrm{C}_{60}$ attachment, due to inter- or intra-molecular interactions. By hydrating these areas, the water molecules disrupt the interactions between these contacts and provide a new contact for attachment, which was previously unavailable. This effect has been illustrated for the sorption of HOCs by a Pahokee peat hydrated by water (Graber and Borisover, 1998) and other polar solvents (Borisover et al. 2001). The results of the current study illustrate a similar effect for 
the attachment of $\mathrm{C}_{60}$ by two different humics, bringing into account more complexity in the environmental fate and transport of $\mathrm{C}_{60}$.

Another possible contributing factor to the observed increase in attachment involves the swelling of the AP-SOM layer. At higher temperatures the AP-SOM layers expand or swell due to increased thermal motions and apparent increased hydration, much like the matrix relaxation observed in previous studies (Schaumann, 2005; Schaumann and LeBoeuf, 2005). Swelling will increase the volume of the layers, allowing them to take a more extended conformation from the surface of the sensor and also provide an increasingly cross-linked or bridged mesh of organic matter for the $\mathrm{C}_{60}$ particles to become entangled in. At lower temperatures, the AP-SOM layers dehydrate, which compacts the layer. This compacted layer likely has a more condensed, less extended (smoother) surface with reduced potential for $\mathrm{C}_{60}$ particle entanglement, effectively lowering attachment efficiency (Fig. 4). This finding is in agreement with a study by Chen and Elimelech (2008) which investigated the deposition of $\mathrm{C}_{60}$ onto an alginate coated silica surface. When the alginate was exposed to a higher electrolyte concentration, the layer compacted and resulted in lower attachment efficiency.

\section{Conclusions}

In this study, we have shown that the attachment of $\mathrm{C}_{60}$ to immobilized layers of HHA and HFA is greatly affected by temperature and that the trend in attachment efficiency onto the AP-SOM layers deviate greatly from that of bare silica. The attachment efficiency onto both AP-SOM layers hinder attachment at low temperatures and then begin to rise as temperatures in- 
crease, eventually resulting in enhanced attachment at high temperatures, in the case of the HHA layer. (Fig. 4). Based on thermal characterization of the AP-SOM layers compared to the corresponding attachment trends (Figs. 5 and 6), the attachment efficiency is strongly correlated with hydration of the layer. Regions of rapid hydration correspond to steep increases in attachment and regions of slowed or decreased hydration correspond to lower increases in attachment. Possible mechanisms explaining this phenomenon include water-assisted disruption of polar SOM contacts and hydration induced swelling of the AP-SOM matrix. This thermal effect on the attachment of $\mathrm{C}_{60}$ to AP-SOM may prove to be crucial in understanding the fate and transport of ENMs in areas that are rich in AP-SOM, like the subsurface environment, because humic substances typically represent the dominant organic components in soils and sediments (Stevenson, 1994). In areas that see large temperature fluctuations throughout the year, we may see a much larger uptake of ENMs in the warm seasons and less uptake in the cold seasons. Understanding how ENMs interact with individual components of soil is crucial to understanding the fate and transport of ENMs in a complex soil system, leading to their eventual impact on ecosystem and human health. The thermal effect illustrated in the current study may also prove useful in the removal of similar ENMs from wastewater or drinking water, by exploiting the much higher attachment efficiency at increased temperatures. Further studies need to be carried out, however, to investigate the relative importance of other variables which may play a role in ENM interactions with AP-SOM. 


\section{Acknowledgements}

The present study was supported by the National Science Foundation Award Nos. 1133528 and 1133280, the Vanderbilt University Discovery Grant Program, and the Vanderbilt University Department of Civil and Environmental Engineering.

\section{References}

G.V. Andrievsky, M.V. Kosevich, O.M. Vovk, V.S. Shelkovsky, and L.A. Vashchenko. On the production of an aqueous colloidal solution of fullerenes. Journal of the Chemical Society-Chemical Communications, (12):1281-1282, 1995.

M. Borisover and E.R. Graber. Relationship between strength of organic sorbate interactions in nom and hydration effect on sorption. Environmental science \& technology, 36(21):4570-4577, 2002.

M. Borisover, M. Reddy, and E.R. Graber. Solvation effect on organic compound interactions in soil organic matter. Environmental science $\&$ technology, 35(12):2518-2524, 2001.

S. Bosi, T. Da Ros, G. Spalluto, and M. Prato. Fullerene derivatives: an attractive tool for biological applications. European Journal of Medicinal Chemistry, 38(11-12):913-923, 2003.

K.L. Chen and M. Elimelech. Aggregation and deposition kinetics of fullerene (c-60) nanoparticles. Langmuir, 22(26):10994-11001, 2006. 
K.L. Chen and M. Elimelech. Influence of humic acid on the aggregation kinetics of fullerene $\left(c_{6} 0\right)$ nanoparticles in monovalent and divalent electrolyte solutions. Journal of Colloid and Interface Science, 309(1):126-134, 2007.

K.L. Chen and M. Elimelech. Interaction of fullerene (c-60) nanoparticles with humic acid and alginate coated silica surfaces: Measurements, mechanisms, and environmental implications. Environmental Science $\&$ Technology, 42(20):7607-7614, 2008.

R.C. DeLapp, E.J. LeBoeuf, J. Chen, and B.H. Gu. Advanced thermal characterization of fractionated natural organic matter. Journal of Environmental Quality, 34(3):842-853, 2005.

E.R. Graber and M. Borisover. Hydration-facilitated sorption of specifically interacting organic compounds by model soil organic matter. Environmental science 8 technology, 32(2):258-263, 1998.

E.R. Graber, L. Tsechansky, and M. Borisover. Hydration-assisted sorption of a probe organic compound at different peat hydration levels: the link solvation model. Environmental science \& technology, 41(2):547-554, 2007.

R. Hogg, T.W. Healy, and D.W. Fuerstenau. Mutual coagulation of colloidal dispersions. Transactions of the Faraday Society, 62:1638-1651, 1966.

N. Ishida and S. Biggs. Direct observation of the phase transition for a poly(n-isopropylacryamide) layer grafted onto a solid surface by afm and qcm-d. Langmuir, 23(22):11083-11088, 2007. 
X. Jiang, M. Tong, H. Li, and K. Yang. Deposition kinetics of zinc oxide nanoparticles on natural organic matter coated silica surfaces. Journal of colloid and interface science, 350(2):427-434, 2010.

J.P. Kamat, T.P.A. Devasagayam, K.I. Priyadarsini, and H. Mohan. Reactive oxygen species mediated membrane damage induced by fullerene derivatives and its possible biological implications. Toxicology, 155(1):5561, 2000.

E.J. LeBoeuf. Macromolecular characteristics of natural organic matter and their influence on sorption and desorption behavior of organic chemicals. University of Michigan, 1998.

E.J. LeBoeuf and W.J. Weber. A distributed reactivity model for sorption by soils and sediments .8. sorbent organic domains: Discovery of a humic acid glass transition and an argument for a polymer-based model. Environmental Science \&3 Technology, 31(6):1697-1702, 1997.

D. Lin, X. Tian, F. Wu, and B. Xing. Fate and transport of engineered nanomaterials in the environment. Journal of environmental quality, 39 (6):1896-1908, 2010.

Y. Lu, K. Yang, and D. Lin. Transport of surfactant-facilitated multiwalled carbon nanotube suspensions in columns packed with sized soil particles. Environmental Pollution, 192:36-43, 2014.

D.Y. Lyon, L.K. Adams, J.C. Falkner, and P.J.J. Alvarez. Antibacterial activity of fullerene water suspensions: Effects of preparation method 
and particle size. Environmental Science \& Technology, 40(14):4360-4366, 2006.

C.G. Malmberg and A.A. Maryott. Dielectric constant of water from 0 to $100{ }^{\circ} \mathrm{C}$. Journal of Research of the National Bureau of Standards, 56(1): $1-8,1956$.

M.S. Mauter and M. Elimelech. Environmental applications of carbon-based nanomaterials. Environmental Science \&6 Technology, 42(16):5843-5859, 2008.

A. McWilliams. Nanotechnology: A realistic market assessment, 2014. URL

http://www.bccresearch.com/market-research/nanotechnology/ nanotechnology-market-assessment-report-nan031f.html.

N. Nakajima, C. Nishi, F.M. Li, and Y. Ikada. Photo-induced cytotoxicity of water-soluble fullerene. Fullerene Science and Technology, 4(1):1-19, 1996.

A. Nel, T. Xia, L. Madler, and N. Li. Toxic potential of materials at the nanolevel. Science, 311(5761):622-627, 2006.

X.L. Qu, P.J.J. Alvarez, and Q.L. Li. Impact of sunlight and humic acid on the deposition kinetics of aqueous fullerene nanoparticles (nc(60)). Environmental Science \& Technology, 46(24):13455-13462, 2012.

G. Sauerbrey. Verwendung von schwingquarzen zur wagung dunner schichten und zur mikrowagung. Z. Phys, 155:206-222, 1959.

G.E. Schaumann. Matrix relaxation and change of water state during hydra- 
tion of peat. Colloids and Surfaces A: Physicochemical and Engineering Aspects, 265(1):163-170, 2005.

G.E. Schaumann and E.J. LeBoeuf. Glass transitions in peat: their relevance and the impact of water. Environmental science 83 technology, 39(3):800806, 2005.

F.J. Stevenson. Humus chemistry : genesis, composition, reactions. Wiley, New York, 2nd edition, 1994.

R. Sutton and G. Sposito. Molecular structure in soil humic substances: the new view. Environmental Science \& Technology, 39(23):9009-9015, 2005.

A. Vidyasagar, C. Sung, R. Gamble, and J.L. Lutkenhaus. Thermal transitions in dry and hydrated layer-by-layer assemblies exhibiting linear and exponential growth. ACS Nano, 6(7):6174-6184, 2012.

M.V. Voinova, M. Rodahl, M. Jonson, and B. Kasemo. Viscoelastic acoustic response of layered polymer films at fluid-solid interfaces: continuum mechanics approach. Physica Scripta, 59(5):391, 1999.

W.J. Weber, E.J. LeBoeuf, T.M. Young, and W.L. Huang. Contaminant interactions with geosorbent organic matter: Insights drawn from polymer sciences. Water Research, 35(4):853-868, 2001.

\section{Appendix A. Details on Experimental Methods}

Appendix A.1. $C_{60}$ stock suspension preparation

First, approximately $35 \mathrm{mg}$ of $\mathrm{C}_{60}$ powder was dissolved in $30 \mathrm{~mL}$ toluene, forming a clear, dark purple solution. Then, $5 \mathrm{~mL}$ of this mixture was intro- 
duced into a beaker containing $50 \mathrm{~mL}$ deionized water, forming two distinct phases. This solution was then sonicated (Fisher Sonic Dismembrator 300, Pittsburgh, PA) for more than 3 hours in a fume hood to allow for evaporation of the toluene. The beaker was topped off with deionized water every 20 minutes to account for the evaporated water. The resulting transparent, brown-yellow liquid was filtered through $0.22 \mu \mathrm{m}$ pore size membrane filter paper (Fisher, GSWP02500), resulting in a clear, bright yellow liquid. This method was repeated a total of 6 times to produce approximately $330 \mathrm{~mL}$ of $\mathrm{C}_{60}$ stock suspension.

\section{Appendix A.2. HHA and HFA solution preparation}

Fifty milligrams of dry HHA was introduced into $400 \mathrm{~mL}$ deionized water and stirred for more than 3 hours. During this time, the $\mathrm{pH}$ was adjusted drop wise with $0.1 \mathrm{~N} \mathrm{NaOH}$ to a $\mathrm{pH}$ of 10.0 to allow the HHA to completely dissolve. After 3 hours and complete dissolution of the HHA, the $\mathrm{pH}$ was adjusted drop wise with $0.1 \mathrm{~N} \mathrm{HCl}$ down to a $\mathrm{pH}$ of 6.7 and filtered through $0.22 \mu \mathrm{m}$ pore size membrane filter paper. The stock solution was then diluted to $500 \mathrm{~mL}$ total volume, including the addition of $\mathrm{NaCl}$ and phosphate buffer to make a $0.1 \mathrm{~g} / \mathrm{L} \mathrm{HHA}, 70 \mathrm{mM} \mathrm{NaCl}$, and $2 \mathrm{mM}$ phosphate buffer solution. Fifty milligrams of dry HFA was introduced into $400 \mathrm{~mL}$ of deionized water and stirred for more than three hours. Once the HFA had completely dissolved, the $\mathrm{pH}$ was adjusted drop wise with $0.1 \mathrm{~N} \mathrm{NaOH}$ to a $\mathrm{pH}$ of 6.7 and filtered through $0.22 \mu \mathrm{m}$ pore size membrane filter paper. The stock solution was then diluted to $500 \mathrm{~mL}$ total volume, including the addition of $\mathrm{NaCl}$ and phosphate buffer, to make a $0.1 \mathrm{~g} / \mathrm{L} \mathrm{HFA,} 70 \mathrm{mM} \mathrm{NaCl}$, and 2 $\mathrm{mM}$ phosphate buffer solution. 
Appendix A.3. $C_{60}$ deposition experimental procedure

The background solution, $70 \mathrm{mM} \mathrm{NaCl}$ and $2 \mathrm{mM}$ phosphate buffer, was flowed over the surface of the sensors until a stable baseline was achieved. The signal was considered stable when the third overtone frequency $\left(f_{3}\right)$ failed to drift more than $0.3 \mathrm{~Hz}$ over the course of 10 minutes. Once a stable baseline was achieved, $2 \mathrm{~mL}$ of a $0.1 \mathrm{~g} / \mathrm{L}$ PLL solution that contained the same electrolyte and buffer concentrations was flowed across the sensor surface. During this time, the PLL adsorbed to the silica surface very rapidly. The PLL layer was then rinsed with $2 \mathrm{~mL}$ of the background solution. If PLL is the final layer before $\mathrm{C}_{60}$ deposition, the layer construction ended here. In the case of the HHA and HFA experiments, a solution of $0.1 \mathrm{~g} / \mathrm{L}$ HHA or HFA, containing the same electrolyte and buffer concentrations, was then flowed across the rinsed PLL layer until the signal once again stabilized, $\approx 20$ minutes. During this time, the oppositely charged HHA or HFA macromolecules readily adsorbed onto the rinsed PLL layer, forming a second layer. The rapid adsorption of HHA or HFA onto the PLL layer usually resulted in a shift in $f_{3}$ of approximately 20 and $22 \mathrm{~Hz}$, respectively. The mass of HHA and HFA deposited onto the sensor surface was calculated to be approximately 411 and $351 \mathrm{ng} / \mathrm{cm}^{2}$, respectively, by the Voigt viscoelastic model (Voinova from proposal). A plot of the model calculated masses can be seen in Figure B.7. Finally, the HHA or HFA layer was then rinsed with $2 \mathrm{~mL}$ of the background solution until a stable baseline was achieved. The layer construction portion of all experiments took place at $25{ }^{\circ} \mathrm{C}$.

Once the layers were constructed, the temperature was adjusted to the desired temperature of the $\mathrm{C}_{60}$ deposition experiment. All solutions used 
in each experiment were held at $2{ }^{\circ} \mathrm{C}$ above the set point temperature of the experiment for several hours before the experiment took place to ensure the solutions would not degas on the surface of the sensor. Once a stable baseline was achieved in the background electrolyte, measured amounts of the stock $1 \mathrm{M} \mathrm{NaCl}$ and $1 \mathrm{M}$ phosphate buffer were added to the diluted stock $\mathrm{C}_{60}$ suspension to make a $\mathrm{C}_{60}$ concentration of $6 \mathrm{mg} / \mathrm{L}$, with electrolyte and buffer concentrations matching all other solutions. The resulting diluted $\mathrm{C}_{60}$ suspension was then immediately flowed into the flow module and across the surface of the sensor. As $\mathrm{C}_{60}$ deposited onto the sensor surface, the mass continuously increased, causing a decrease in frequency (Eq. 1). The first 120 seconds of this drop in frequency were used to calculate the initial deposition rates for each set of experimental conditions. 
479 480

\section{Appendix B. Details on Materials Characterization}

Appendix B.1. Harpeth humic and fulvic acid characterization details

Table B.1: Elemental analysis and water content of HHA and HFA after DeLapp et al. (2005), reproduced here with permission from the author.

\begin{tabular}{|c|c|c|c|c|c|c|c|}
\hline NOM & $\begin{array}{c}\mathrm{C} \\
(\mathrm{mass} \\
\%)\end{array}$ & $\begin{array}{c}\mathrm{H} \\
(\mathrm{mass} \\
\%)\end{array}$ & $\begin{array}{c}\mathrm{O} \\
(\mathrm{mass} \\
\%)\end{array}$ & $\begin{array}{c}\mathrm{N} \\
(\mathrm{mass} \\
\%)\end{array}$ & $\begin{array}{c}\mathrm{S} \\
(\mathrm{mass} \\
\%)\end{array}$ & $\begin{array}{c}\mathrm{P} \\
(\mathrm{mass} \\
\%)\end{array}$ & $\begin{array}{c}\text { Water } \\
\text { Content } \\
\text { (mass \%) }\end{array}$ \\
\hline $\begin{array}{c}\text { Harpeth } \\
\text { Humic } \\
\text { Acid }\end{array}$ & 51.2 & 4.9 & 39.1 & 4.0 & 0.56 & 0.12 & 5.8 \\
\hline $\begin{array}{c}\text { Harpeth } \\
\text { Fulvic } \\
\text { Acid }\end{array}$ & 43.4 & 3.4 & 49.4 & 1.5 & 0.20 & 0.75 & 5.2 \\
\hline
\end{tabular}


Table B.2: Relative abundance of functional groups by ${ }^{13} \mathrm{C}$ nuclear magnetic resonance (NMR) analysis of HHA and HFA after DeLapp et al. (2005), reproduced here with permission from the author.

\begin{tabular}{|c|c|c|c|c|c|}
\hline NOM & $\begin{array}{c}\text { Alkyl } \\
0-50 \\
(\mathrm{ppm})\end{array}$ & $\begin{array}{c}\text { O-Alkyl } \\
50-112 \\
(\mathrm{ppm})\end{array}$ & $\begin{array}{c}\text { Aromatic } \\
112-145 \\
(\mathrm{ppm})\end{array}$ & $\begin{array}{c}\text { Phenolic } \\
145-163 \\
(\mathrm{ppm})\end{array}$ & $\begin{array}{c}\text { Carboxyl } \\
163-190 \\
(\mathrm{ppm})\end{array}$ \\
\hline $\begin{array}{c}\text { Harpeth } \\
\text { Humic } \\
\text { Acid }\end{array}$ & 0.2 & 0.28 & 0.22 & 0.13 & 0.18 \\
\hline $\begin{array}{c}\text { Harpeth } \\
\text { Fulvic } \\
\text { Acid }\end{array}$ & 0.15 & 0.29 & 0.19 & 0.18 & 0.20 \\
\hline
\end{tabular}

The mass of the layers formed by depositing HHA and HFA were determined to be approximately 411 and $351 \mathrm{ng} / \mathrm{cm}^{2}$, respectively, by the Voigt viscoelastic model (Voinova et al., 1999). A plot of the model calculated masses can be seen in Figure B.7. The AP-SOM layers were further characterized thermally, to ensure that the high temperatures did not facilitate desorption of the AP-SOM, which would in turn affect $\mathrm{C}_{60}$ deposition. After the layers were constructed, as described in Appendix A.3, they were taken through a temperature ramp which encompassed the entire temperature range of the experiment. Upon cooling the AP-SOM layers, the frequency curve returned to its original value in each case, meaning the mass of the layers remained unchanged and desorption was not observed. Representative heating/cooling curves for HHA and HFA can be seen in Figures B.8 and B.9, respectively. 


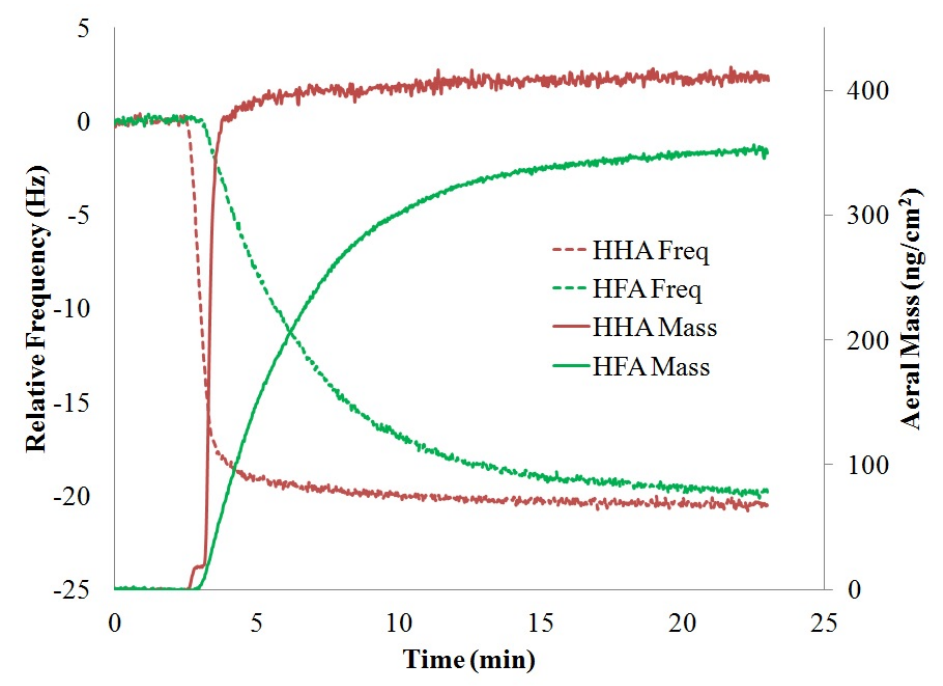

Figure B.7: Calculated mass of the HHA and HFA layers, by the Voigt viscoelastic model.

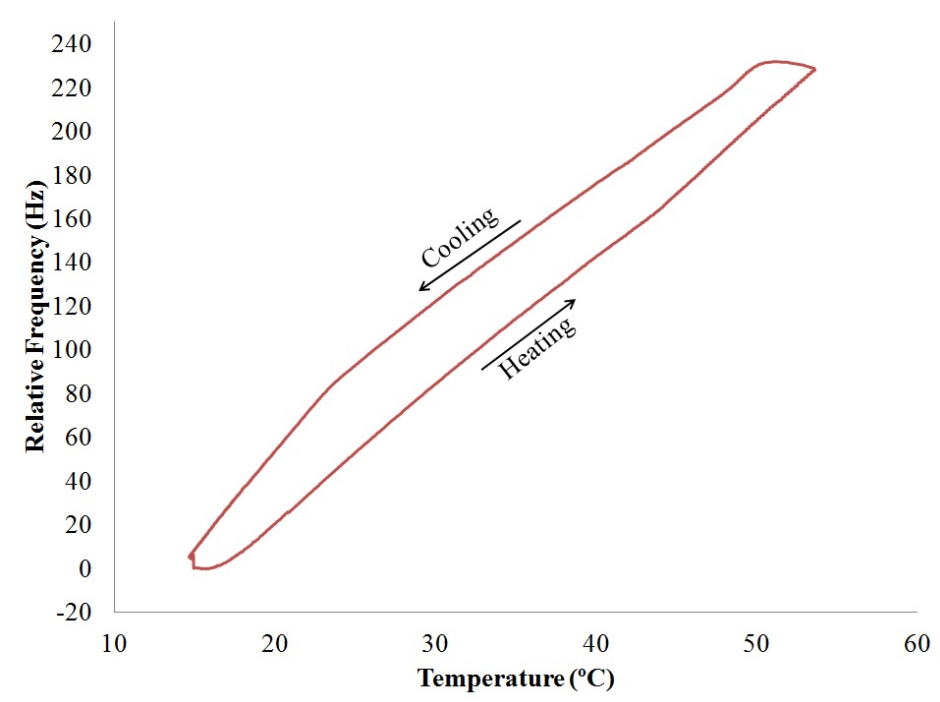

Figure B.8: The heating/cooling loop of HHA, showing no change in mass over the temperature range investigated. 


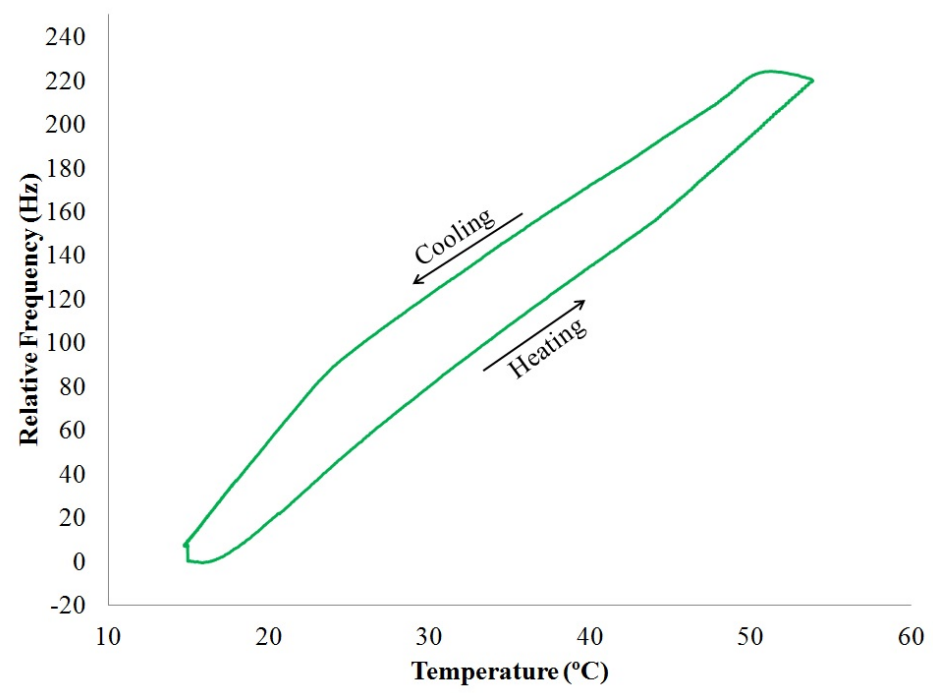

Figure B.9: The heating/cooling loop of HFA, showing no change in mass over the temperature range investigated.

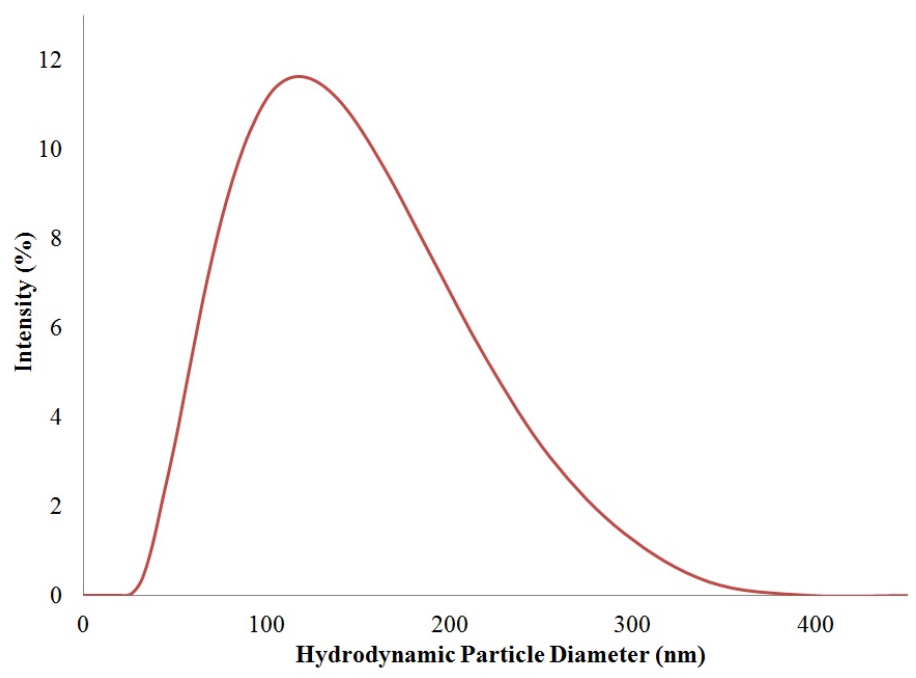

Figure B.10: Particle size distribution of the $\mathrm{C}_{60}$ nanoparticle stock suspension by DLS, based on 14 independent measurements. 


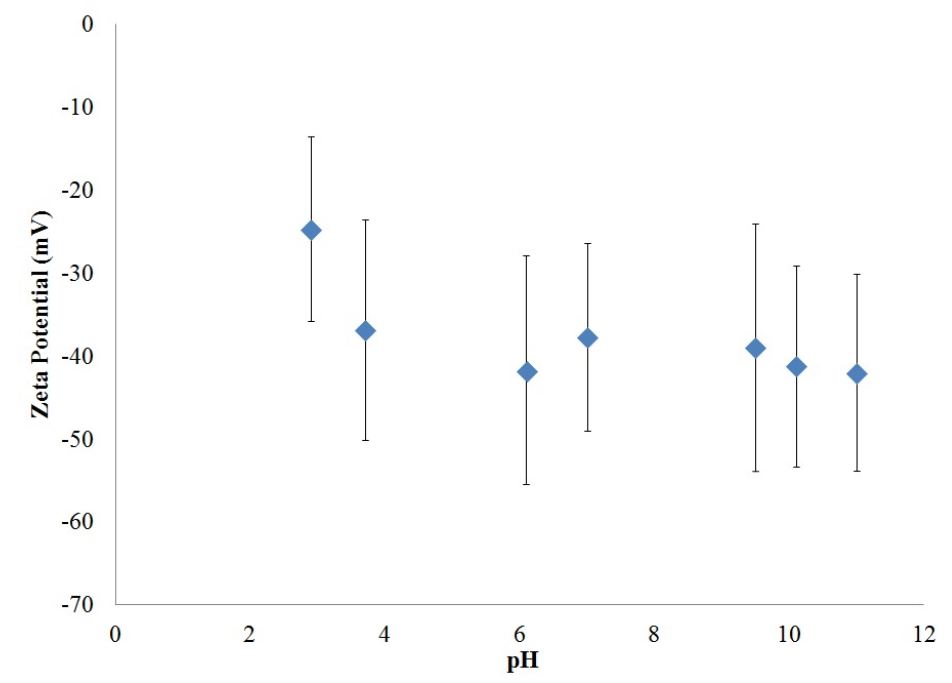

Figure B.11: Zeta potential of the $\mathrm{C}_{60}$ nanoparticle stock suspension over a $\mathrm{pH}$ range of 310, by DLS. Each point represents 14 independent measurements and error bars represent a $95 \%$ confidence interval.

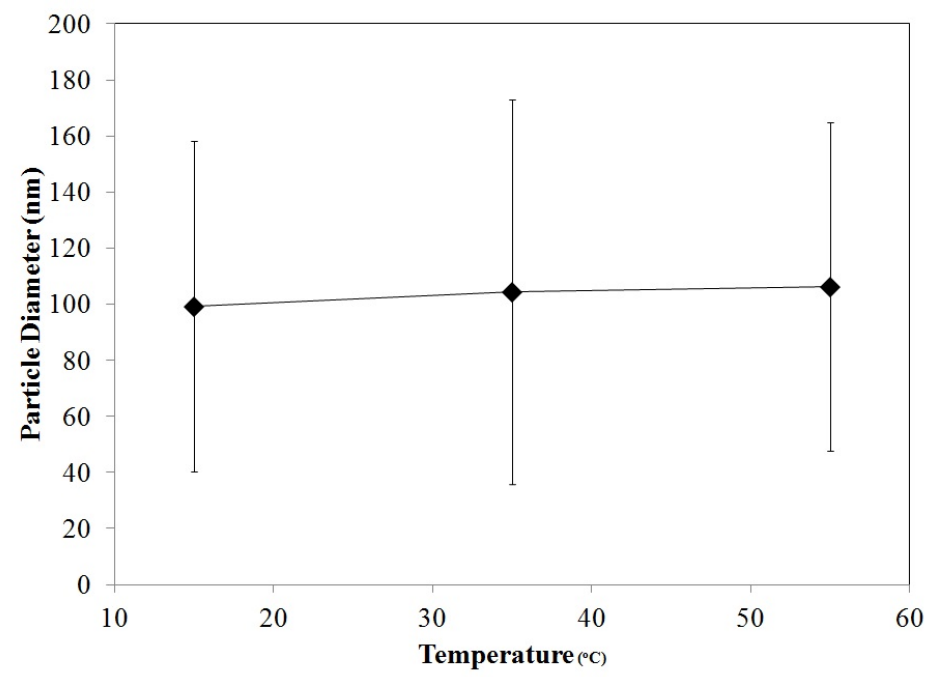

Figure B.12: Hydrodynamic particle diameter of the $\mathrm{C}_{60}$ stock suspensions as a function of temperature. Each point represents 20 independent measurements and error bars represent standard deviation. 


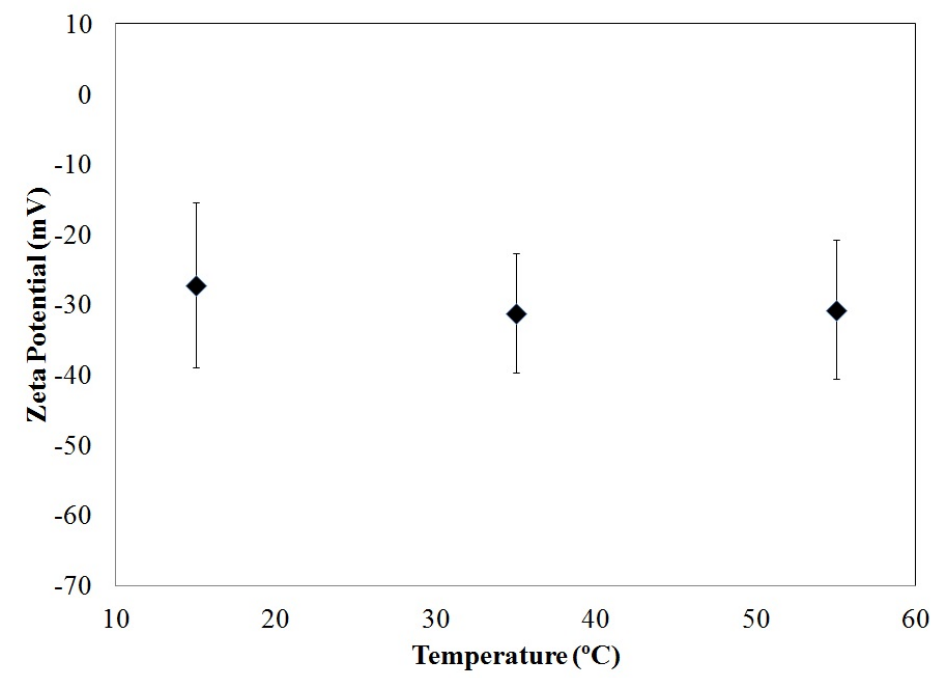

Figure B.13: Zeta potential of the $\mathrm{C}_{60}$ stock suspension as a function of temperature. Each point represents 14 independent measurements and error bars represent the standard deviation.

\section{Appendix C. DLVO interaction energy calculation details}

By treating the $\mathrm{C}_{60}$ deposition as a sphere-plate interaction, the total interaction energy between the $\mathrm{C}_{60}$ particles and the silica sensor surface was estimated from the sum of the electrical double layer repulsion $\left(V_{R}\right)$ and van der Waals attraction $\left(V_{A}\right)$. Eq. C.1 (Hogg et al., 1966) was used to calculate the van der Waals attraction, where $A$ is the combined Hamaker constant for the $\mathrm{C}_{60}$ water silica system $\left(4.71 \times 10^{-21} \mathrm{~J}\right)$ Qu et al. 2012), $a$ is the radius of $\mathrm{C}_{60}$ particles (Table C.3), $h$ is the separation distance between the $\mathrm{C}_{60}$ particle and silica sensor surface, and $\lambda$ is the characteristic wavelength of interaction (usually assumed to be $100 \mathrm{~nm}$ ) (Jiang et al., 2010).

$$
V_{A}=-\frac{A a}{6 h}\left(1+\frac{14 h}{\lambda}\right)^{-1}
$$


Eq. C.2 Hogg et al., 1966) was used to calculate the electrical double layer repulsion, where $\epsilon_{0}$ is the dielectric permittivity in a vacuum, $\epsilon_{r}$ is the relative dielectric permittivity of water (Table C.3), $\zeta_{p}$ is the zeta potential of the $\mathrm{C}_{60}$ particles $(-27.2 \mathrm{mV}$, remained constant over the temperature range investigated according to DLS measurements), $\zeta_{c}$ is the zeta potential of the silica surface $(-30 \mathrm{mV})$, and $\kappa$ is the inverse Debye length.

$$
V_{R}=\pi \epsilon_{0} \epsilon_{r} a\left\{2 \zeta_{p} \zeta_{c} \ln \left[\frac{1+\exp (-\kappa h)}{1-\exp (-\kappa h)}\right]+\left(\zeta_{p}^{2}+\zeta_{c}^{2}\right) \ln [1-\exp (-2 \kappa h)]\right\}
$$

Eq. C.3 was used to calculate the inverse Debye length for the electrolyte system (Jiang et al., 2010), where $e$ is the elementary charge, $n_{j}$ is the number concentration of ions in the bulk solution, $z_{j}$ is ion valence, $k$ is the Boltzmann constant, and $T$ is the absolute temperature.

$$
\kappa=\sqrt{\frac{e^{2} \sum n_{j} z_{j}^{2}}{\epsilon_{0} \epsilon_{r} k T}}
$$

Since the $\mathrm{C}_{60}$ particle radius and relative dielectric permittivity of water vary with temperature, the values used in Eqs. C.1, C.2, and C.3 are summarized in Table C.3. The values for the relative dielectric permittivity of water were obtained from a study by Malmberg and Maryott (1956). 
Table C.3: Temperature-dependent $\mathrm{C}_{60}$ particle radius and relative dielectric permittivity of water used to calculate the total interaction energy between the $\mathrm{C}_{60}$ particles and silica sensor surface.

\begin{tabular}{|c|c|c|}
\hline Temperature $\left({ }^{\circ} \mathrm{C}\right)$ & $\mathrm{C}_{60}$ Particle Radius $(\mathrm{nm})$ & $\epsilon_{r}$ \\
\hline 15 & 49.7 & 81.95 \\
\hline 25 & 50.8 & 78.30 \\
\hline 35 & 52.2 & 74.83 \\
\hline 45 & 52.5 & 71.51 \\
\hline
\end{tabular}

